\title{
A new concept for the treatment of brain cancer
}

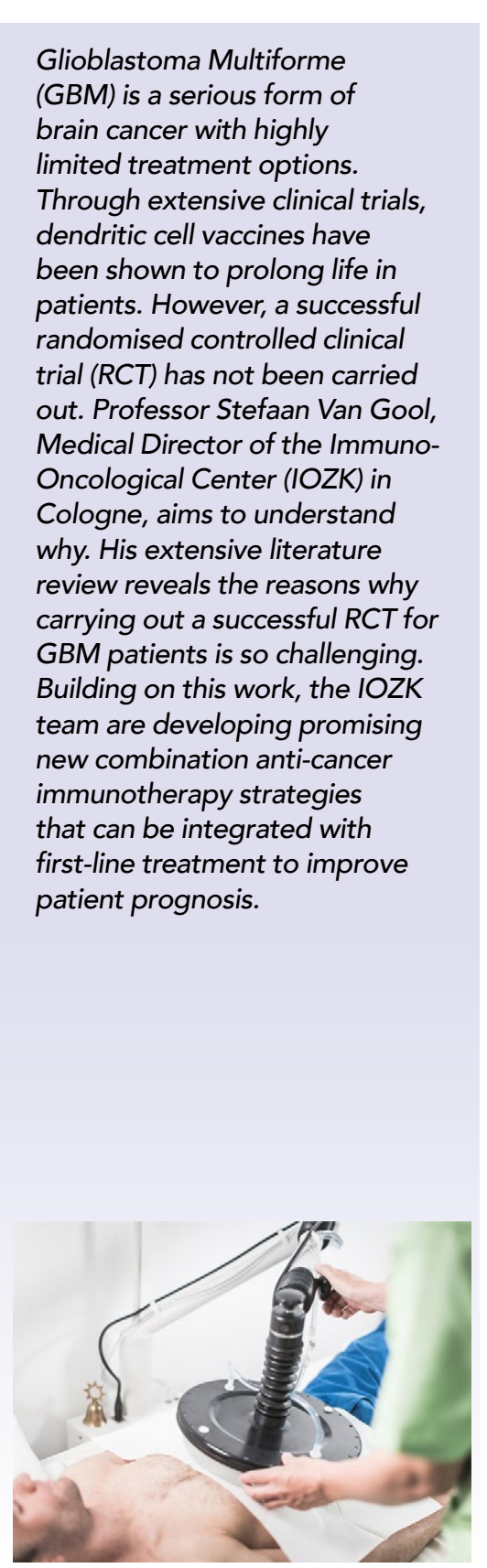

ancer is the second leading cause
of human death worldwide, accounting for one in every six deaths. Cancer is the subject of and potential cures. There are many forms of serious cancer, but one of the most difficult to treat is Glioblastoma. Multiforme (GBM). There is a need for improved treatments for GBM patients, like the inclusion of first-line immunotherapy to fight their cancers Meta-analyses (that is, the examination of data from many independent studies, to help determine overall trends) have investigated the effectiveness of specific immunotherapy treatment for GBM. These studies show that immunotherapy can cause a significant increase in surviva time. However, no single research group has successfully demonstrated the efficacy controlled trial (RCT).

Professor Stefaan Van Gool, Medical Director at the Immuno-Oncologica Center (IOZK) in Cologne, has led extensive analysis of the literature to to better understand why this is the case. His team's findings provide evidence highlighting multiple issues that arise when assessing GBM immunotherapy treatments via RCTs. Building on their studies, the IOZK team is developing active and specific immunotherapies that, in combination with other anticancer therapies, provide exciting therapeutic potential.

\section{WHAT MAKES GBM SO SERIOUS?} Brain tumours are the leading causes of cancer deaths in males aged 20-39 and the fourth most common cause in females of the same age. However of cancer, and the most frequent malignant brain cancer in adults. It is a rapidly growing tumour of the brain and spinal cord, which causes symptoms to progress swiftly. The years of loss of life due to GBM is the highest of all cancers, in part due to its occurrence at a young age and its poor prognosis. Despite this, GBM is referred to as an orphan disease as it is rare and has not had major interest from drug companies in developing a treatment. The current standard-of-care following diagnosis of GBM includes neurosurgery, radiochemotherapy, and maintenance chemotherapy. Unfortunately, however these treatments do not fully control its poor prognosis.

\section{TREATING GBM WITH} IMMUNOTHERAPY

Unfortunately, the available options for treating people with GBM have not changed in recent years. However, research has been carried out into innovative new areas of treatment including tumour-treating fields, anti-angiogenic treatments, targeted therapies, oncolytic virus therapy, and immunotherapies.

One example of a targeted mmunotherapy is dendritic cell (DC) vaccines. Dendritic cells help the immune system to recognise and attack To make the vaccine, scaintists grols. To make the vaccine, scientists grow

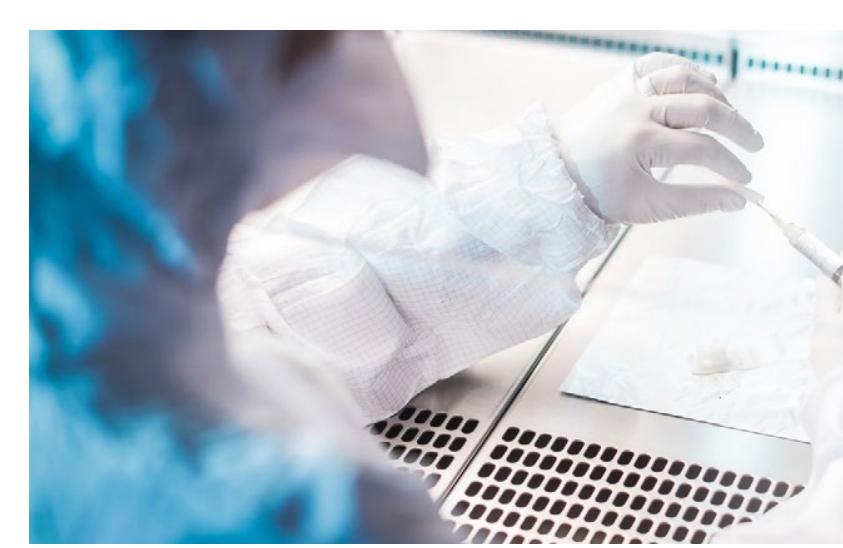

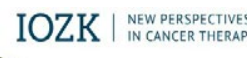

own defence system, to help fight their cancer:

antigens in the lab. The vaccine then system to attack the cancer

Systematic reviews of phase I and II trials have shown a significant improvement five years) when DC vaccines were administered to people with GBM The effectiveness of DC vaccines for GBM reached a level 2a and level $1 \mathrm{c}$ of evidence (Oxford Centre for Evidence-Based Medicine). So why has the neurooncology community not succeeded in conducting an RCT? Professor Van Gool's extensive investigation of the erature highlights several reasons why

\section{HOW DO RANDOMISED}

\section{WORK?}

Well-designed RCTs that are conducted properly can provide a useful

comparison of different treatments. $\mathrm{RCTs}$ $m$ to allow for the factors that are not directly under experimental control.

In a clinical context, RCTs are used to compare the effects of different drugs, medical devices, surgical techniques, treatments. Individuals enlisted in RCTs are differ crols onlisted in both known and unknown ways. These

OZK are developing active and specific mmunotherapies that, in combination

with other anti-cancer therapies,

vide exciting therapeutic potential.

differences can't be directly controlled, but they can influence study outcomes. By randomly allocating people between the treatments being compared, an RCT (hes researchers staistical control over

RCTs are considered to be the most reliable form of scientific verification in heltharchy of evidence that inluences they reduce spurious causality and bias. In some areas, great progress has been made through RCTs. The trials tend to work by comparing a new

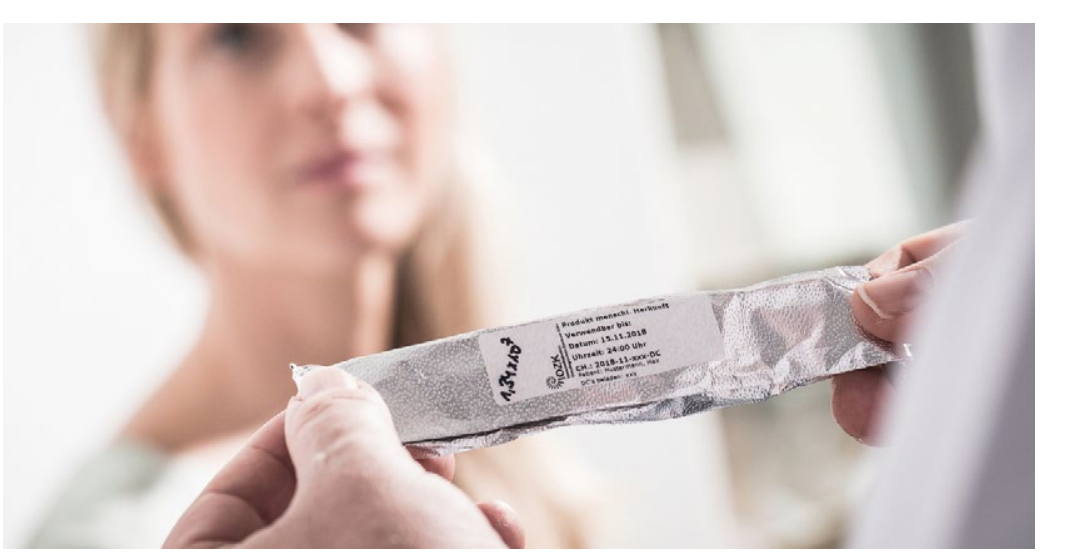

\section{GBM IMMUNOTHERAPIES?} Professor Van Gool carried out a review of the literature around how good RCTs are at assessing the efficacy of are many reapy. He concluded that there can be problematic.

Firstly, the design of an RCT can be a major challenge. It can be hard to find an appropriately matched control group as the clinical stans of the patient, the tumour biology, and chemosensitivity (MGMT promoter methylation status) of

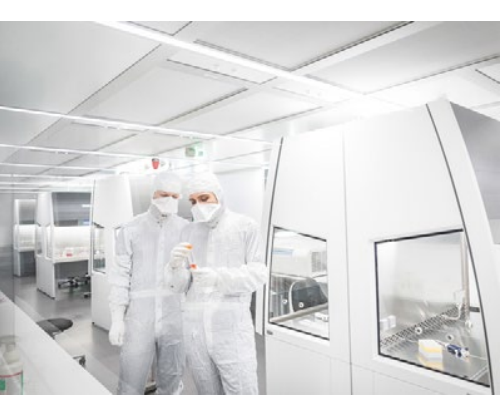

Unfortunately, the avilable options
octrteating people with GBM have 


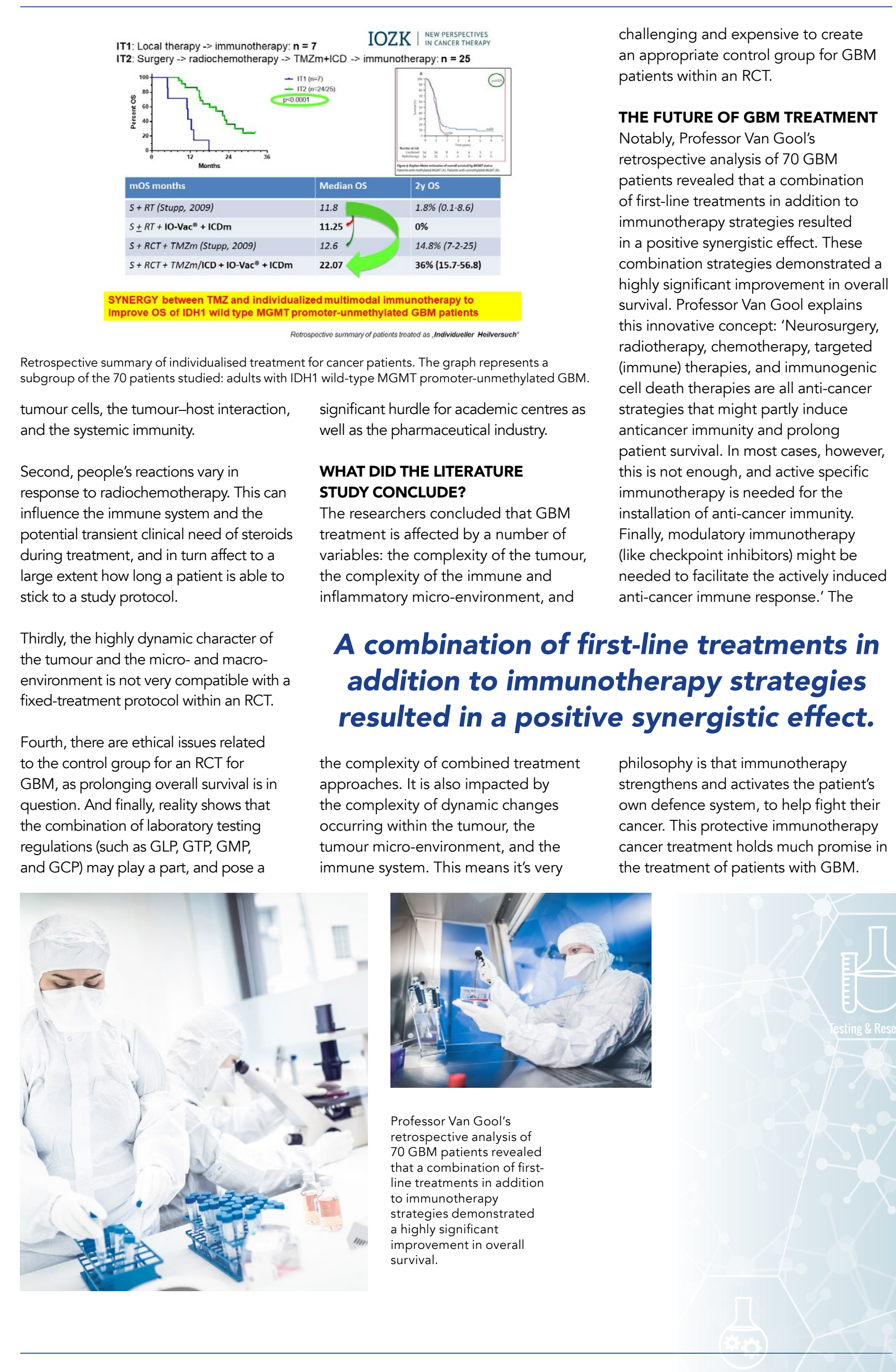

\section{Research Objectives}

The Immuno-Oncological Center (IOZK), a private nonprofit organisation in Cologne, delivers individualised

multimodal immunotherapy for cancer patients.

\section{Detail}

Address

IOZK. Hohenstaufenring 30-32

50674 Köln, Germany

Bio

Stefaan Van Gool, MD, PhD, is a paediatric neuro-

oncologist. After his studies in Leuven and Münster, he became Professor and successfully established dendritic cell vaccination for brain cancer in Europe. In 2015 he Center Köln where he delivers individualised multimod immunotherapy for cancer patients.

IOZK | $\mid \begin{aligned} & \text { Immun-OnKologisches } \\ & \text { zentrum köLn }\end{aligned}$

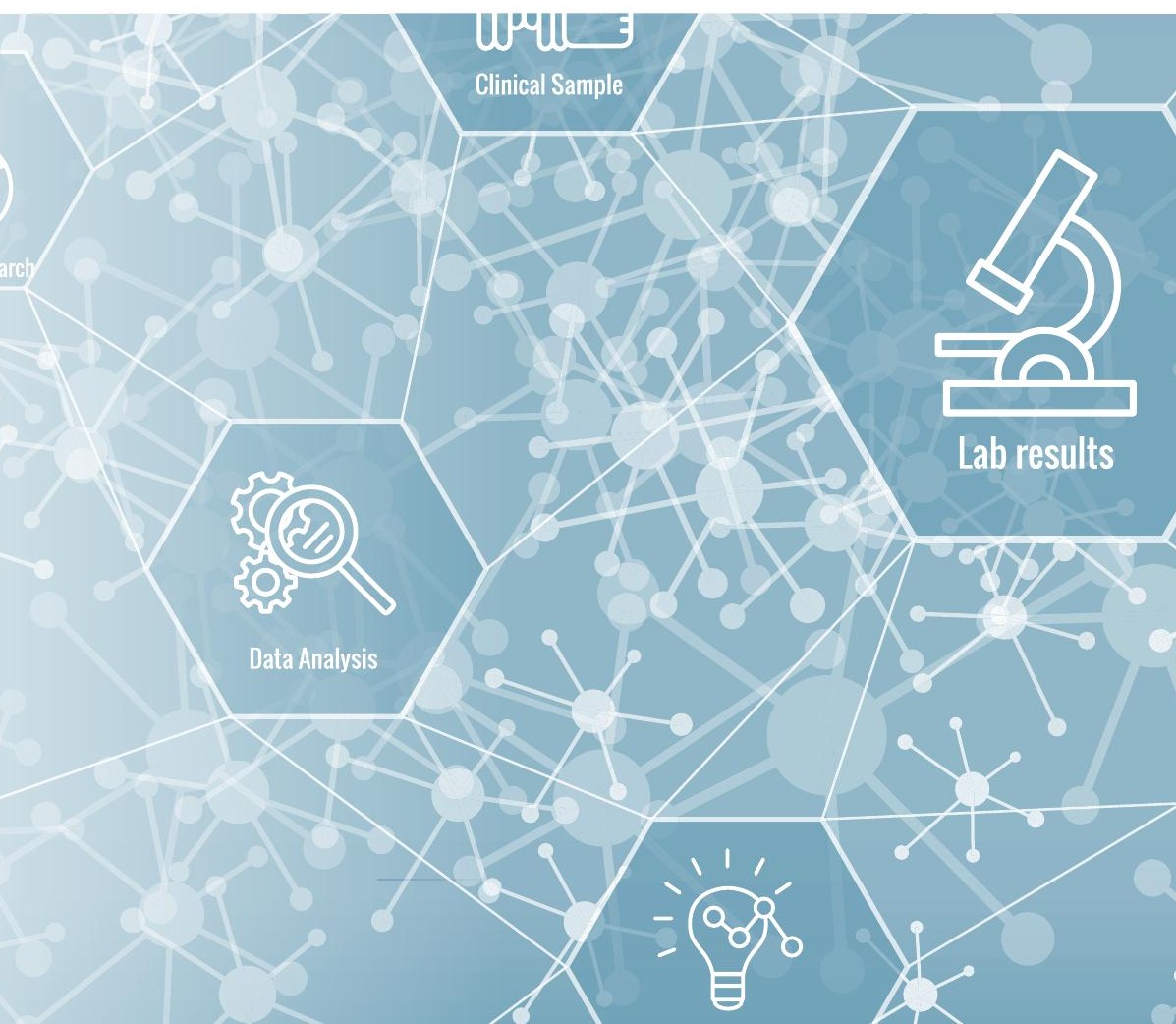

\section{References}

Van Gool, SW, (2021) Randomised Controlled Immunotherapy Clinical Trials for GBM Challenged. Cancers. 13(1), 32. doi. org/10.3390/cancers 13010032

Sprenger, T, Schirrmacher, V, Stücker, W, Van Gool, SW, (2020) Position paper: new insights into the immunobiology and dynamics of tumor-host interactions require adaptations 20(8).639-646. do: 10.1080/147371402020.1785874

Galluzzi, L, Vitale, I, Warren, S, Adjemian, S, et al, (2020) Consensus guidelines for the definition, detection and interpretation of immunogenic cell death. Jo 10 116/jitc-2019 $\underline{000337}$

Van Gool, SW, Makalowski, J, Feyen, O, et al, (2018) The Induction of Immunogenic Cell Death (ICD) During Maintenance Chemotherapy and Subsequent Multimodal Immunotherapy for Glioblastoma (GBM). Austin Oncol Case Rep, 3(1): 1010

\section{Personal Response}

How close are we to bringing multimodal immunotherapy into routine practice for GBM patients? II Clinically and technically, I would say 'very close'. The treatment is ambulant and does not add any major toxicity. Patients can be referred to a few specialised centres in
each country where this part of the treatment can be administered, in close communication with the local neuro-oncologists from many countries together to build up a network within Europe for delivering DC vaccination. However, at the regulatory and health insurance level, I have to say 'very far'. All must work together to pass these hurdles, with the goal of prolonging life, and good
quality of life, for patients with GBM. 